\title{
COVID-19 lockdowns in low- and middle-income countries: Success against COVID-19 at the price of greater costs
}

South Africa (SA) received international acclaim, including from the World Health Organization (WHO), for the speed and severity of its lockdown response to the SARS-CoV-2 pandemic that continues to wreak havoc on the health, economic and social front worldwide. Missing from this narrative is the negative impact of this response on SA's already precarious health system and fragile economy that has catapulted households into poverty, spawned job losses and unharnessed a wave of gender-based violence. Lockdown strategies have been implemented by many highly resourced Northern Hemisphere countries to contain the pressure on healthcare facilities battling to cope with a massive influx of COVID-19 cases when the epidemic was peaking. Lockdowns have also being precipitously adopted by many sub-Saharan African (SSA) countries, albeit at a much earlier stage of the regional epidemic than in the North. SA was among the first in SSA to embark on a lockdown, at a stage when the majority of COVID-19 cases were diagnosed in travellers and there were few sporadic community transmission cases.

The rationale for embarking on a total and highly restrictive lockdown in SA, with partial relaxation recently, included slowing the effective reproductive rate and aspiring to virus containment. However, an efficient testing strategy for COVID-19, inclusive of ability to trace at least $70 \%$ of contacts, which is vital to achieve containment, was not in place. ${ }^{[1]}$ Shortcomings of the testing strategy for COVID-19 case identification in SA at the time of the start of the level 5 lockdown included restrictive criteria on who to test, and inadequate capacity to test at scale by the National Health Laboratory Service (NHLS) and in the private sector. The NHLS is the sole laboratory service provider to all SA public health facilities, which provide care to $\sim 80 \%$ of the population. The NHLS plan to scale up SARS-CoV-2 molecular-based tests to 36000 per 24 hours by the end of April 2020 has still not materialised, ${ }^{[2]}$ partly owing to lack of preparedness, and dependence on importation of testing materials that are in high demand leading to massive global shortages. Consequently, SA's ability to contain the virus floundered.

The number of confirmed COVID-19 cases in SA increased 10-fold during the lockdown, from an initial $30-40$ per day in the first fortnight when $<1000$ daily tests were conducted, to 400 per day as the number of daily tests also increased 10 -fold. ${ }^{[3]}$ As of 17 June 2020, the National Institute for Communicable Diseases reported $>4000$ newly diagnosed cases in the past 24 hours alone, ${ }^{[4]}$ despite the modest rate of testing (20.3 per 1000 population), compared with testing rates in high-income countries ( $>100$ per 1000 population). The number of confirmed COVID-19 cases in Africa on 17 June 2020 was 270660 with 72490 deaths, with SA being the country with the highest number of COVID-19 cases $(n=80412 ; 29.7 \%)$ and deaths $(n=1674 ; 23.1 \%) .{ }^{\left[{ }^{[}\right]}$ The higher numbers in SA may at least partly be due to its greater capacity than other SSA countries (e.g. 0.5 per 1000 in Nigeria) to conduct molecular-based testing to confirm COVID-19 diagnosis, because of prior investment in HIV viral load monitoring and tuberculosis (TB) diagnostics. This rate of testing, however, lags behind smaller nations such as Mauritius (119.7 per 1000 population). ${ }^{[5]}$ The SA lockdown may have transiently reduced the rate of SARS-CoV-2 community transmission, delayed the surge of severe COVID-19 cases, and provided opportunity for better healthcare facilities preparedness.

Other SSA countries that also embarked on lockdowns at an early stage of their epidemics had even less testing capacity than SA to diagnose active COVID-19 cases, very much negating lockdown as a strategy for virus containment in most of SSA, as identification of infectious cases and tracing of their contacts are integral to achieving containment. ${ }^{[1]}$ If the pandemic is not mitigated, the WHO estimates that deaths from COVID-19 in SSA (population 1078 billion) during 2020 could range from 83000 to $190000,{ }^{[6]}$ while modelling from SA (population 59.2 million) suggests that there may be 48000 deaths within the next 4 months in SA alone, ${ }^{[7]}$ reflecting major uncertainties of the modelling upon which life-threatening decisions are possibly being made.

In 2018, the WHO estimated that $25 \%$ of 1.45 million deaths from TB occurred in SSA, with 66000 of these deaths in SA (44 000 in people living with HIV and 22000 in HIV-negative people)..$^{[8]}$ Modelling indicates that if the COVID-19 pandemic led to a $25 \%$ global reduction in expected TB detection for 3 months, it could increase TB deaths by $13 \% .{ }^{[9]}$

Using data from the NHLS, the sole laboratory service provider to the public sector, we analysed for trends in testing for suspected $\mathrm{TB}$ and diagnosis of microbiologically confirmed $\mathrm{TB}$, immediately prior to the level 5 lockdown, during level 5 and during the subsequent period when transitioning to level 4 . Segmented linear regression models are fitted to daily accounts, which include a term for time, indicator variables for level 5, level 4 and level 3 lockdown periods and time since 27 March 2020, 1 May 2020 and 1 June 2020 (when level 5, level 4 and level 3 lockdowns began, respectively). The fitted models are plotted alongside daily counts to show abrupt changes in trend (rate of change in testing or results) and level (immediate increase or decrease at time of lockdown implementation) post lockdown.

In $\mathrm{SA}$, testing for $\mathrm{TB}$ decreased by $50 \%$, from a weekly average of 49109 tests during the 7 weeks pre-lockdown to an average of 24620 during the 5-week level 5 lockdown period, and remained at this rate through level 4 lockdown. Concurrently the weekly average of microbiologically confirmed TB cases declined by 33\% from a weekly average of 3707 to 2465 between the pre-lockdown and level 5 lockdown period and remained as such during level 4 lockdown (Fig. 1). This significant reduction in TB diagnosis is likely to result in delay of initiation of TB treatment and threatens to reverse gains made in reducing the incidence of TB in SA, and lends itself to exacerbating Mycobacterium tuberculosis transmission to others. The disruption of TB services represents but a single example of the unintended consequences of an unmeasured approach such as total lockdown to mitigate the consequences of the COVID-19 pandemic.

SA has the largest HIV treatment programme in the world, with steady progress to the UNAIDS $90-90-90$ targets, including a $40 \%$ reduction in new HIV infections in 2019 compared with 2010. ${ }^{[10]}$ During the level 5 lockdown, there has been a $22 \%$ reduction in average weekly HIV-1 viral load testing (measured annually in people living with HIV to monitor HIV antiretroviral treatment) done by the NHLS and a 33\% reduction in CD4+ cell count testing (done mainly when initiating antiretroviral treatment), compared with the prelockdown periods (Fig. 2). These decreases suggest possible treatment interruptions, and missed appointments due to patient fear of SARS$\mathrm{CoV}-2$ infection and violating travel restrictions in the context of widespread security force excesses. During level 4 lockdown, weekly averages of HIV-1 viral load and CD4+ testing were only $5 \%$ and $15 \%$ lower compared with pre-lockdown averages, respectively. 
The potential impact that an excessive and poorly implemented response to COVID-19 may have by interrupting of essential motherchild services in low-income countries could indirectly lead to 235500 - 1157000 additional child deaths over a 6-month period, ${ }^{[11]}$ despite children being at extremely low risk for severe COVID-19 illness or death.

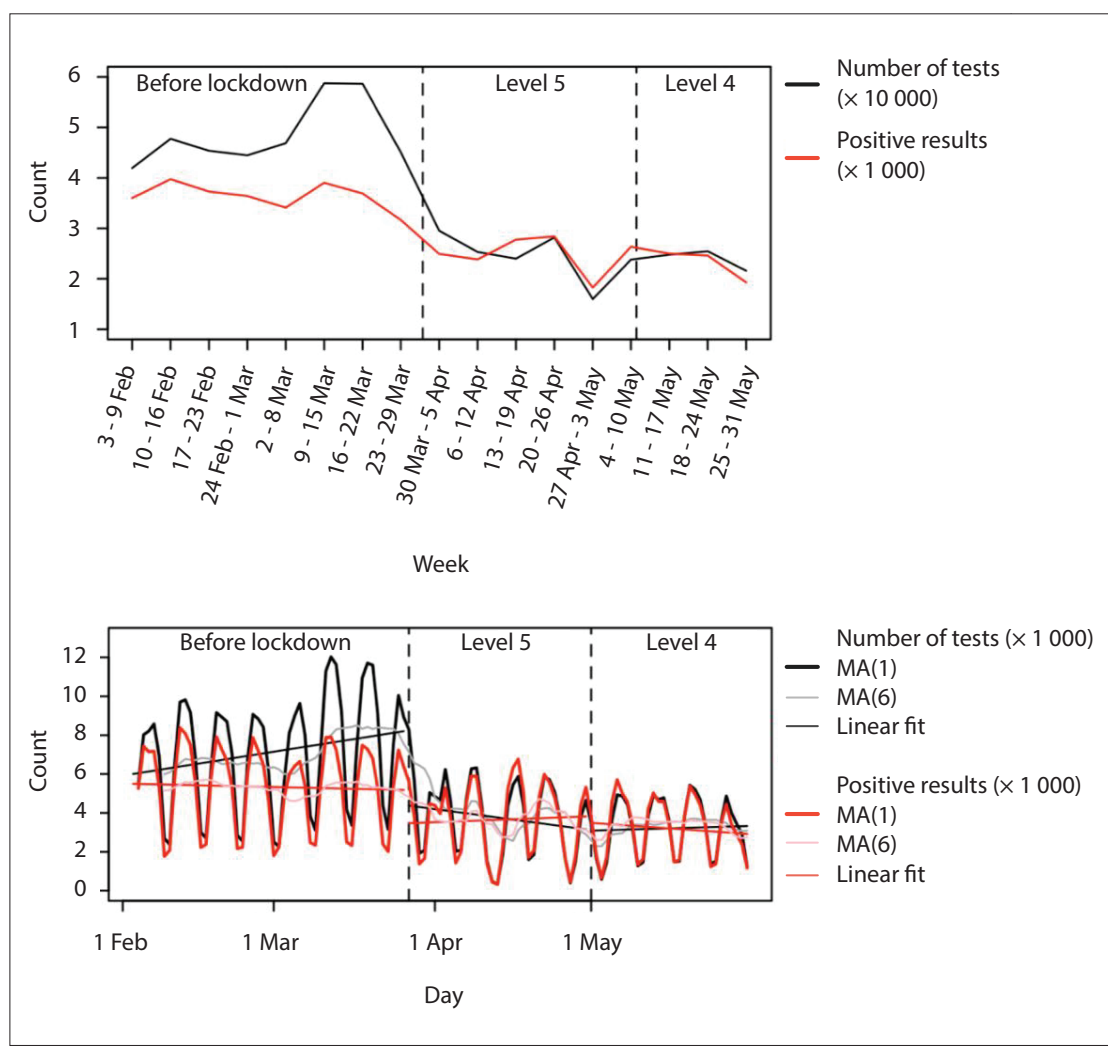

Fig. 1. Weekly and daily totals (presented as a moving average of order 1, MA(1) and order 6, MA(6)) for number of GeneXpert tests administered and cases with Mycobacterium tuberculosis detected done before lockdown, at the time of total lockdown (level 5) and with easing of lockdown (level 4).
The extended lockdown in SA was estimated to have reduced tax revenue collection by a tectonic ZAR285 billion, ${ }^{[12]}$ in contrast to the ZAR200 billion annual budget in 2020/21 for public health that is almost entirely funded off this tax base. Also, the highly restrictive lockdown placed the livelihoods of people in jeopardy, with a high percentage of vulnerable families likely to have been pushed into poverty, and potentially increasing susceptibility to death from other diseases associated with poverty that could exceed the direct number of deaths from COVID-19.

While SSA and SA are unlikely to be spared from deaths due to the COVID-19 pandemic, uncalculated measures such as extended lockdown, while possibly being able to mitigate some effects of the COVID19 pandemic, could, albeit inadvertently, indirectly result in non-COVID-19 morbidity and mortality that could far exceed the 196000 deaths the WHO estimates would occur from COVID-19 in SSA. As evident from early experience with $\mathrm{TB}$ and HIV in SA, we are clearly heading down such a pathway unless the response to COVID-19 is adequately calibrated and essential services are safeguarded. We believe that a strategy focused primarily on COVID-19 can no longer be ethically or morally justified, and will have long-lasting health, societal and economic impacts that may reverberate for decades to come. Rather, more pragmatic approaches are warranted, including emphasis on community mobilisation and

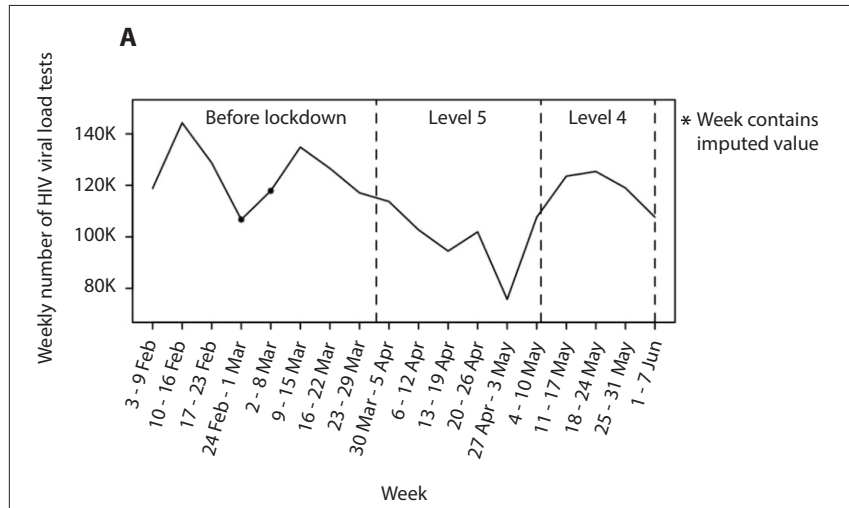

C

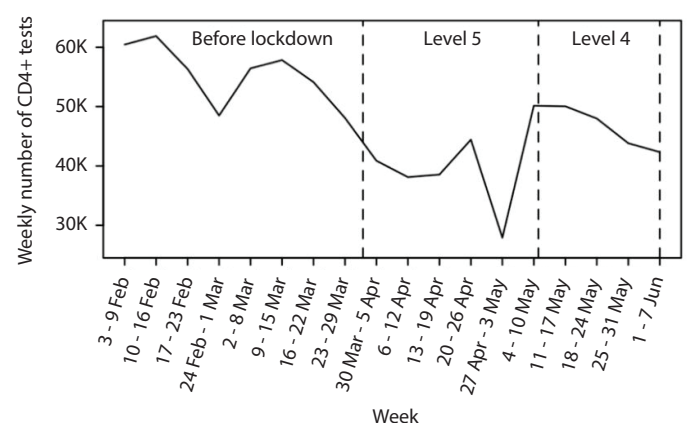

B

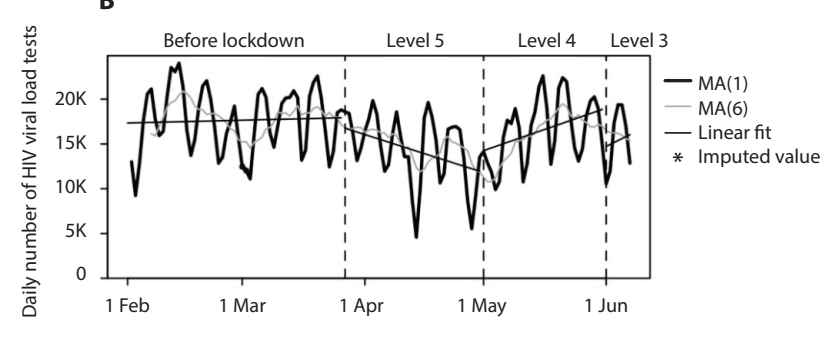

D

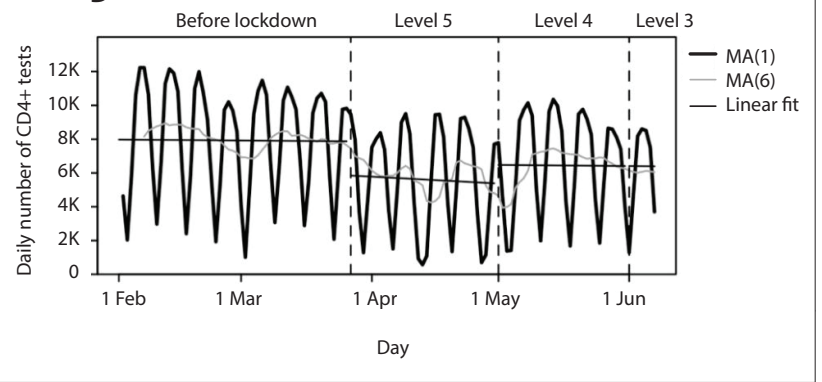

Fig. 2. Number of HIV-1 viral load tests ( $A$ and B) and CD4+ lymphocyte count tests ( $C$ and D) done before lockdown, at the time of total lockdown (level 5) and with easing of lockdown (level 4). 
education on COVID-19, and the widespread adoption of nonpharmaceutical interventions such as use of cloth masks, hand hygiene, and avoiding overcrowded settings.

\section{Shabir A Madhi}

University of the Witwatersand, Johannesburg, South Africa

\section{Glenda E Gray}

South African Medical Research Council, Cape Town, South Africa glenda.gray@mrc.ac.za

\section{Nazir Ismail}

National Institute for Communicable Diseases, Johannesburg, South Africa

\section{Alane Izu}

University of the Witwatersrand, Johannesburg, South Africa

\section{Marc Mendelson}

Groote Schuur Hospital and University of Cape Town, South Africa

\section{Naseem Cassim, Wendy Stevens}

Molecular Medicine and Haematology, University of the Witwatersrand, Johannesburg, South Africa; and National Health Laboratory Service, Johannesburg, South Africa

\section{Francois Venter}

University of the Witwatersrand, Johannesburg South Africa
1. Hellewell J, Abbott S, Gimma A, et al. Feasibility of controlling COVID-19 outbreaks by isolation of cases and contacts. Lancet Glob Health 2020;8(4):e488-e496. https://doi.org/10.1016/S2214$109 \times(20) 30074-7$

2. National Health Laboratory Service. NHLS' preparedness for testing to meet COVID-19 demands 25 March 2020. https://www.nhls.ac.za/nhls-preparedness-for-testing-to-meet-covid-19-demands (accessed 15 May 2020).

3. News24. COVID-19 in South Africa. https://specialprojects.news24.com/sa-coronavirus-statistics index.html (accessed 15 May 2020).

4. National Institute for Communicable Diseases. National COVID-19 daily report. https://www.nicd. ac.za/wp-content/uploads/2020/06/COVID19-Daily-Report-National-Public-17June2020.pdf (accessed 18 June 2020).

5. Worldometer. COVID-19 coronavirus pandemic. https://www.worldometers.info/coronavirus/ \#countries (accessed 18 June 2020).

6. World Health Organization. New WHO estimates: Up to 190000 people could die of COVID-19 in Africa if not controlled. 7 May 2020. https://www.afro.who.int/news/new-who-estimates-190-000people-could-die-covid-19-africa-if-not-controlled (accessed 18 June 2020).

7. Actuarial Society of South Africa. Actuarial Society's COVID-19 model confirms govcernment's risk concerns. 29 April 2020. https://www.actuarialsociety.org.za/download/press-release-29april-2020/ (accessed 18 June 2020).

8. World Health Organization. Global Tuberculosis Report 2019. https://apps.who.int/iris/bitstream/ handle/10665/329368/9789241565714-eng.pdf?ua=1 (accessed 18 June 2020).

9. World Health Organization. Tuberculosis and COVID-19. 11 May 2020. https://www.who.int/tb/ COVID 19 considerations tuberculosis services.pdf (accessed 18 June 2020).

10. UNAIDS. New modelling research shows partial progress in South Africa's response to HIV. 28 June 2019. https://www.unaids.org/en/resources/presscentre/featurestories/2019/june/20190628_south-
UNAIDS. New modelling research shows partial progress in South Africa's response to HIV. 28 June africa-modelling (accessed 18 June 2020).

11. Roberton T, Carter ED, Chou VB, et al. Early estimates of the indirect effects of the COVID-19 pandemic on maternal and child mortality in low-income and middle-income countries: A modelling study. Lancet Glob Health 2020;8:e901-e908. https://doi.org/10.1016/S2214-109X(20)30229-1

12. ANA News. SA tax agency faces R285 bln revenue shortfall due to Covid-19. 5 May 2020. https: $/$ www africannewsagencycom/economy/SA-tax-agency-faces-R285-bln-revenue-shortfall-due-toCovid-19-24902034 (accessed 15 May 2020).

S Afr Med J 2020;110(8):724-726. https://doi.org/10.7196/SAMJ.2020.v110i8.15055 